\title{
Computed Tomography of the Petrous Bone: Particularities in Children
}

\author{
Dounia Basraoui, Kenza Elatiqi*, Hicham Jalal \\ Mother and Child Radiology Department, Mohamed VI University Hospital, Marrakesh, Morocco \\ Email: *kenza.ela tiqi@gmail.com
}

How to cite this paper: Basraoui, D., Elatiqi, K. and Jalal, H. (2018) Computed Tomography of the Petrous Bone: Particularities in Children. Advances in Molecular Imaging, 8, 15-24.

https://doi.org/10.4236/ami.2018.82002

Received: April 1, 2018

Accepted: April 27, 2018

Published: April 30, 2018

Copyright (C) 2018 by authors and Scientific Research Publishing Inc. This work is licensed under the Creative Commons Attribution International License (CC BY 4.0).

http://creativecommons.org/licenses/by/4.0/

\section{(c) (i) Open Access}

\begin{abstract}
Like any organ in children, the ear has particular anatomical features that are well shown in imaging. The petrous bone computed tomography (CT) is a valuable tool for diagnosing ear pathologies and evaluating surgical possibilities. In children, the ear has the peculiarity of having the morphology and size of adults, and the only difference resides in the components of the middle and inner ear related to the growth of the temporal bone and the state of ossification which are progressive with age. Some aspects of growth can simulate pathology and must be known. The pneumatisation of the temporal bone occurs gradually after birth and in several outbreaks until adulthood. The external auditory canal, the internal auditory meatus and the petromastoid canal progressively reach the adult aspect because of the growth of the petrous bone. This work aims to highlight the particularities of the petrous bone CT in pediatrics, since it has become widely used in the exploration of malformations, trauma, infectious complications of the ear and in the assessment of deafness.
\end{abstract}

\section{Keywords}

Petrous Bone, Children, CT, Ear Development

\section{Introduction}

Like any organ in children, the ear has some special anatomical features that are well translated in imaging. The petrous bone CT is a valuable tool, allowing a diagnosis approach of ear pathologies and the evaluation of surgical possibilities, but it is also known for its complexity. In children, the ear has the particularity of having the morphology and size of adults, and the only difference resides in the components of the middle and inner ear related to the growth of the temporal bone and the state of ossification which are progressive with age. Some as- 
pects of growth can simulate pathology and must be therefore well known by radiologists [1].

\section{Material and Method}

We retrospectively reviewed all petrous bone CTs performed in children during one year (from January 2017 to December 2017) regardless the indication. Then, a global review of the literature was conducted on Pubmed and Clinical Key using the following keywords in both French and English: temporal bone, child, radioanatomy of the petrous bone, ear development. Articles were selected based on relevance, date of publication, and access opportunities.

\section{Results}

During this period, we collected and reviewed 61 petrous bone's CT performed on children ( 32 females and 29 males). The age varied between 47 days and 14 years. Main channels and sutures of the temporal bone and the different stages of the pneumaticization of tympano-mastoid cavities were recognized in all cases, the pericochlear hypodensities were found in 2 cases and no case of a central hypodensity of the ossicles of the middle ear was observed.

\section{Discussion}

\subsection{Main Channels and Sutures of the Temporal Bone}

The anatomy of the pediatric skull is challenging, and the stages of its evolution are not easily recalled by radiologists. This complexity stems from the variable nature and changing appearances of sutures over the normal developmental period. The distinction between normal sutural anatomy and fractures is critical, not only when known traumatic events alert the radiologist to potential injury, but also in cases of nonaccidental head injury, which carries a significant overall mortality rate of $20 \%$ and leaves $34 \%$ of survivors with severe disability. In these patients, in whom trauma may not be suspected initially, delayed diagnosis further worsens prognosis [2].

Many channels and sutures are situated on the temporal bone and must be distinguished from fractures if trauma context, among which we site:

\subsubsection{Squamosal Suture}

The squamosal suture is composed of the temporosquamosal (Figure 1) and sphenosquamosal (Figure 2) sutures. Historically, these two sutures have occasionally been referred to as separate structures, although they are more typically viewed as two distinct aspects of a single suture. The temporosquamosal suture can be difficult to visualize along its entirety because its usual orientation may be in the axial plane. The temporosquamosal suture arches posteriorly from the pterion (where the frontal, parietal, temporal, and sphenoid bones meet) and connects the squama of the temporal bone with the lower border of the parietal bone. It is continuous posteriorly with the short and nearly horizontal parietomastoid suture, which unites the mastoid process of the temporal bone 


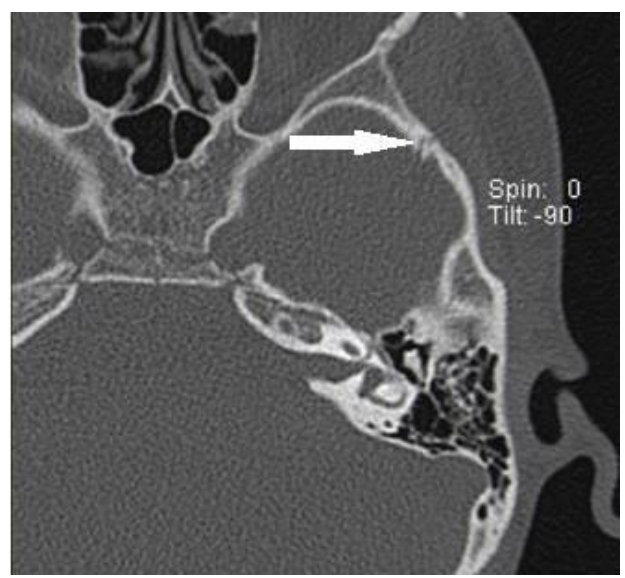

Figure 1. The temporosquamosal suture.

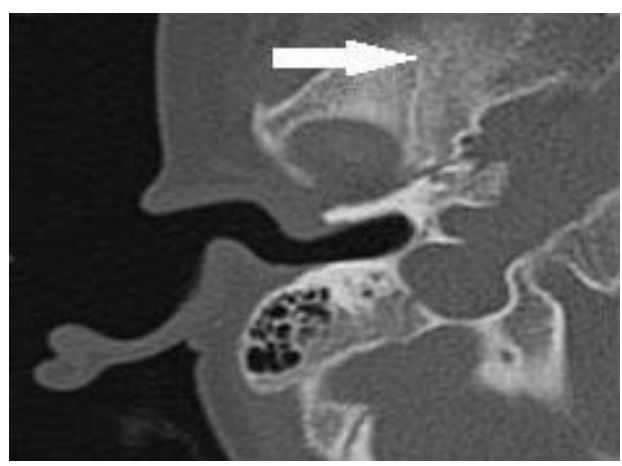

Figure 2. The sphenosquamosal suture.

with the region of the mastoid angle of the parietal bone. The sphenosquamosal suture, which courses inferiorly from the pterion and separates the sphenoid bone from the squama of the temporal bone, is often mistaken for a skull base fracture because of its location [2].

\subsubsection{Occipitomastoïd Suture (Figure 3)}

The occipitomastoid suture extends between the occipital bone and mastoid process of the temporal bone. This suture is a continuation of the lambdoid suture and extends inferiorly to the base of the skull. Not infrequently, the occipitomastoid suture may be mistaken for a skull base fracture, particularly if axial images demonstrate obliquity to the true axial plane so that symmetric visualization of the contralateral suture is hampered. Knowledge of the superior continuation of the occipitomastoid suture, along with the presence of a second symmetric occipitomastoid suture at the base of the skull, are helpful in differentiating this developmental suture from a fracture [2].

\subsubsection{Skull Base Sutures}

The skull base is a difficult area to assess because, at birth, its anterior aspect is largely cartilaginous. Ossification begins in the roof of the ethmoidal labyrinth laterally and spreads toward the midline. By 6 months of age, 50\% of the anterior skull base is completely ossified. This percentage steadily increases, and, by 24 


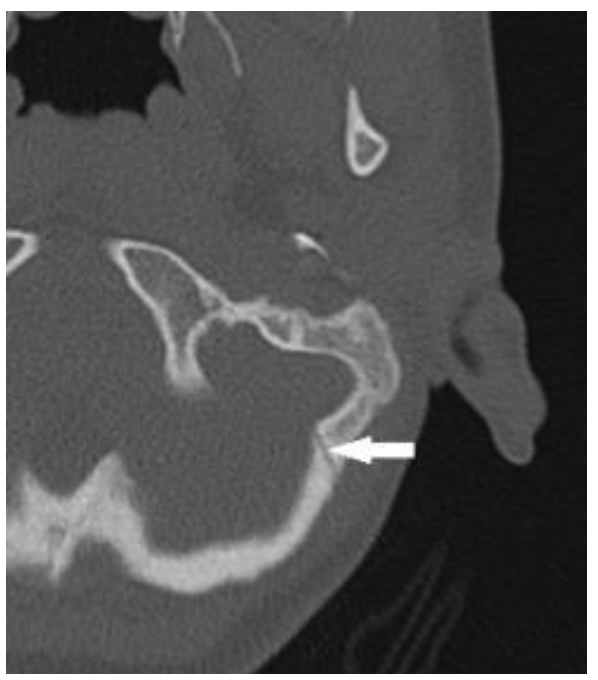

Figure 3. The occipitomastoid suture.

months, $84 \%$ of the anterior skull base is completely ossified. The skull base consists of a number of sutures and synchondroses. The sutures include the innominate (or intraoccipital), lambdoid, occipitomastoid, parietomastoid, and temporosphenoidal sutures. Symmetry and the knowledge of the anatomic appearances of the basal sutures are important for avoiding misdiagnosis. The innominate suture closes by 4 years of age and can be mistaken for a skull base fracture in young children. In this context, the use of re-formatted images obtained oblique to the true axial plane can be beneficial [2].

\subsubsection{The Cochlear Acqueduc (Figure 4)}

The cochlear aqueduct is a bony channel which contains the fibrous periotic duct and connects the peri-lymphatic space of the basal turn of the cochlea with the subarachnoid space of the posterior cranial cavity [3].

The Petro-Tympanic Fissure (Figure 5), the Morganicanal and the Venous Channel Emissary (Figure 6) [1].

\subsection{Peri Cochlear Hypodensities}

From birth up to 4 - 5 years, some peri cochlear hypodensity can persist significantly and may simulate otosclerosis (very rare in children) or osteogenesisimperfecta. It is due to a delayed ossification of the endochondral layer of the otic capsule [4].

Hypoattenuated foci in the otic capsule are routinely depicted with high-resolution CT of the temporal bone in children and have been attributed to either incompletely mineralized bone or cartilage. Controversy exists in radio-logic literature concerning this terminology, because only a few studies have established direct correlation between histology and imaging of the otic capsule [5].

\subsubsection{Hypodensity in the Region of Fissula ante Fenestram (Figure 7)}

The fissula ante fenestram is probably the most common hypoattenuated focus of the otic capsule. The hypodensity is seen in front of the oval window, linear, 


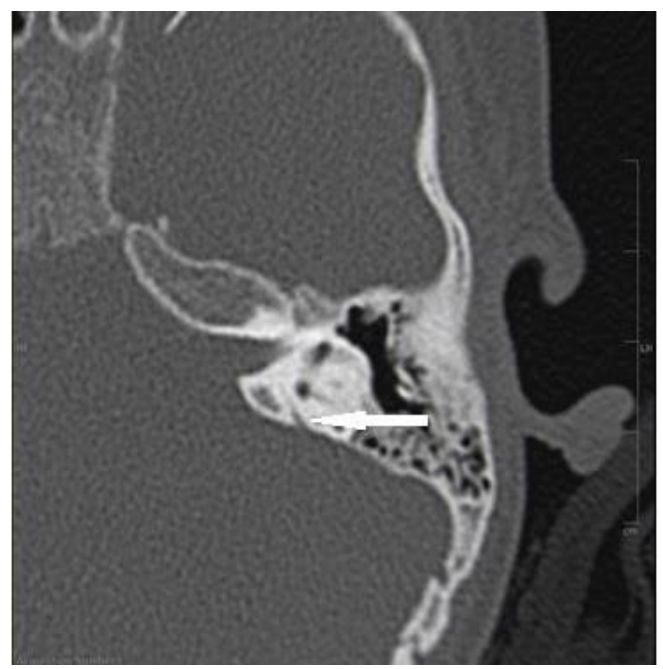

Figure 4. The cochlear acqueduc.

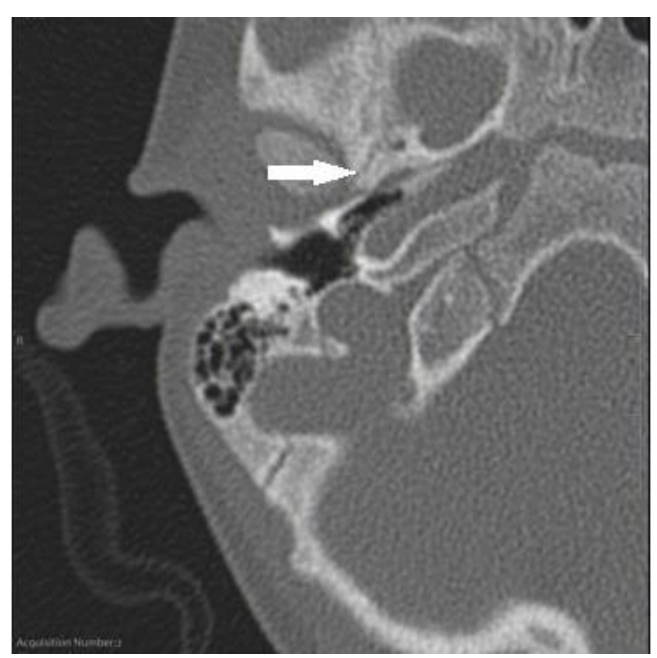

Figure 5. The petro-tympanic fissure.

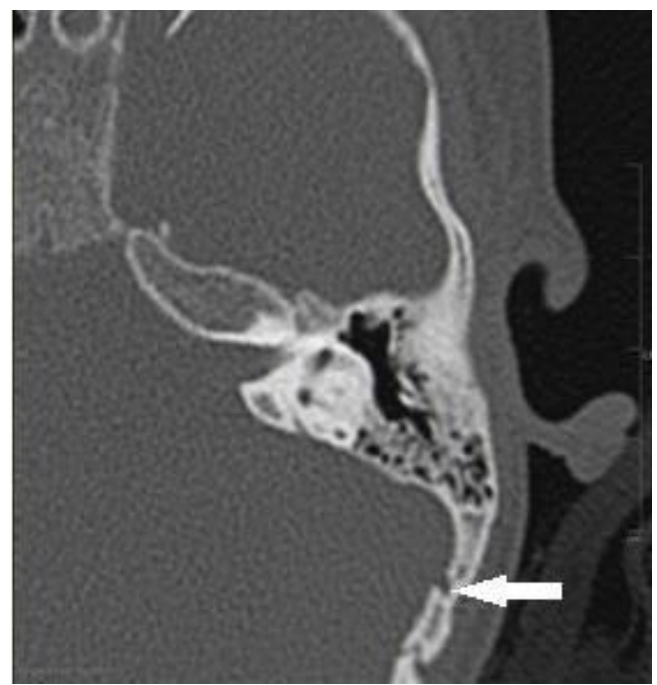

Figure 6. The venous channel emissary. 


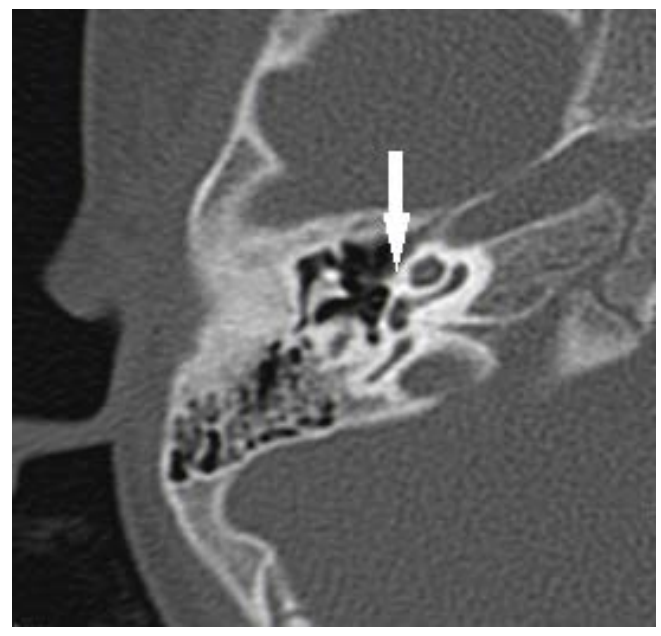

Figure 7. Hypodensity in the region of fissula ante fenestram in a 3 years old child.

parallel to the curve of the cochleain a coronal plane [4]. It is due to a normal presence of fibrocartilage in the area of the fissula ante fenestram, whereas the middle otic layer mainly consisted of cancellous bone [5].

\subsubsection{Focal Hypodensity of the Petrous Apex}

It connects the middle layer of the capsule to the petro-occipital fissure while the petrous apex is not yet developed. It is systematically associated with other hypodensities of the medium capsule. The hypoattenuated focus elongates progressively during its growth. But it still not possible to precise the specific process and ossification centers involved in the growth of the petrous apex. However, the particular location of this hypoattenuated focus, its sequential modifications, and the presence of a cartilaginous cap in its medial aspect suggest that it could be involved in the growth process of the petrous apex. The hypoattenuated focus incidentally observed in children and young adults was restricted to the immediate vicinity of the middle otic layer and could, therefore, represent a remnant from the development of the petrous apex [5].

\subsubsection{Cochlear Cleft}

It corresponds to a small nonosseous space in the otic capsule. On CT, it appears as a linear slot, parallel to the basal turn of the cochlea, located in the middle part of the otic capsule, close to the fissula ante fenestram. It is probable that this cleft is a space between the endosteal and outer periosteal layers of the otic capsule or that it is closely related to the fissula ante fenestram. The cochlear cleft is seen less often with advancing age [6].

\subsection{The Ossicles of the Middle Ear}

The ossicles reach their final size at birth but can present a central hypodensity corresponding to the hematopoietic marrow until the age of 25 months [5]. It seems that the bone marrow space disappeared somewhat earlier in the malleus 
than in the incus. Although the clinical significance of postnatal residual bone marrow within the ossicles is not known, it possibly plays a role as a blood-forming organ in early infancy [7].

\subsection{Pneumaticization of Tympano-Mastoid Cavities (Figure 8)}

There is no agreement as to when pneumatization occurs. Some claim that pneumatized cells can be seen at 24 weeks of gestation, while others state that they are present at birth. Microscopically, an air cell is lined by a single flat layer of epithelium separated from bone by subepithelial connective tissue. The epithelium and connective tissue constitute the mucous membrane of the air cell. According to Wittmaack, the activity of this subepithelial layer is largely responsible for air-cell formation. Radiographically (and macroscopically) air cells are not visible until after birth. The development of air cells is preceded by the formation of bone cavities, a normal physiologic process related to penosteal activity. The bone cavities contain primitive bone marrow, which dedifferentiates into a loose mesenchymal connective tissue. After the epithelial mucous membrane has invaginated, it in turn undergoes atrophy, leaving a thin residual lining membrane attached to the periosteum. "Recession" of the lining membrane and subepithelial bone resorption then further enlarge air cells, but apparently only in the presence of air [8].

The development of complete adult pneumatization can be divided into three stages: the infantile, from birth to 2 years of age; the transitional, from 2 to 5 years; and thereafter the adult [8]. At birth, the middle ear is more or less ventilated but the antrum is not very visible [1]. In the infantile stage, air cells begin to appear and are readily visible by 2 years. In the transitional stage, the squamomastoid undergoes gradual enlargement, with migration of air cells toward the periphery. Once the adult stage is attained, pneumatization ceases [8].

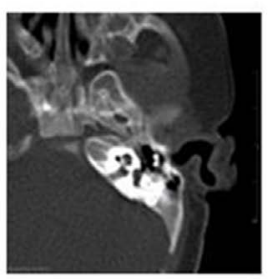

47 days

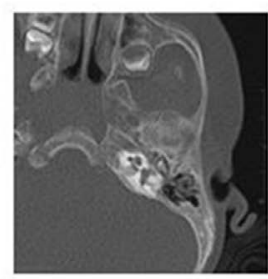

1 year

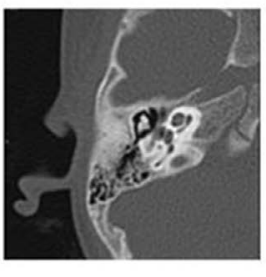

3 year

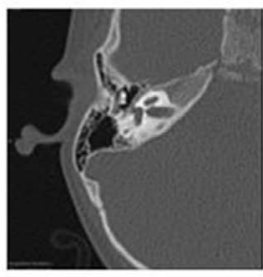

5 year

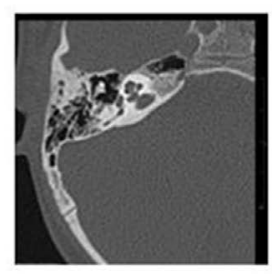

10 year

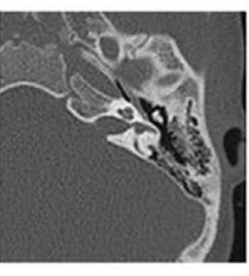

11 year

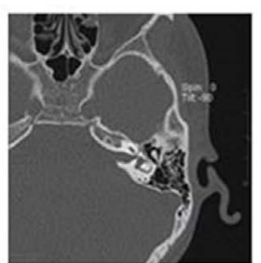

13 year

Figure 8. Pneumaticization of tympano-mastoid cavities. 


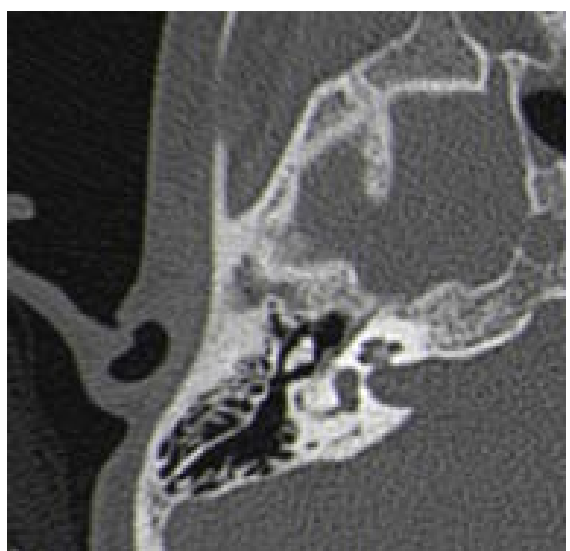

(a)

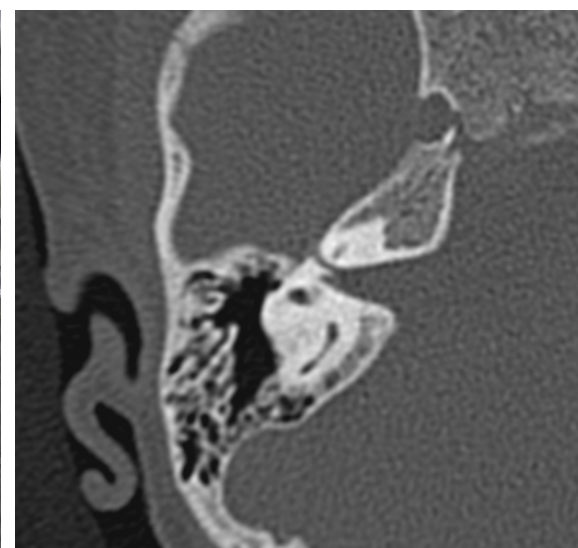

(b)

Figure 9. Internal auditory meatus in a 14 year old child (a) and a 3 years old child (b).

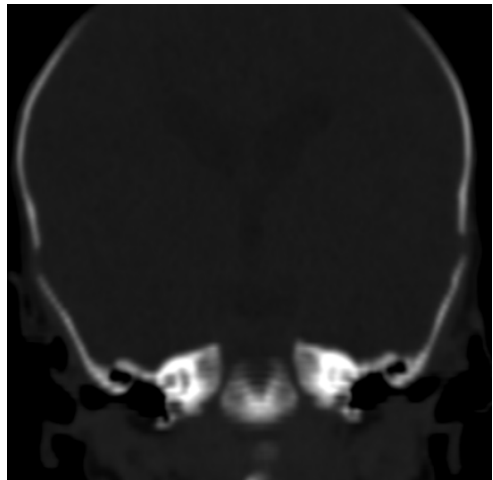

Figure 10. External auditory meatus in a 7 days old newborn.

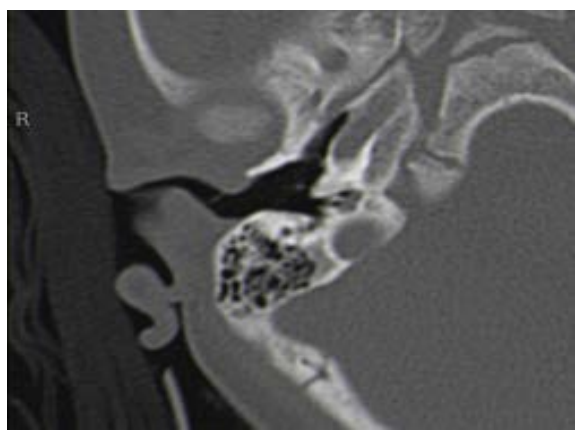

(a)

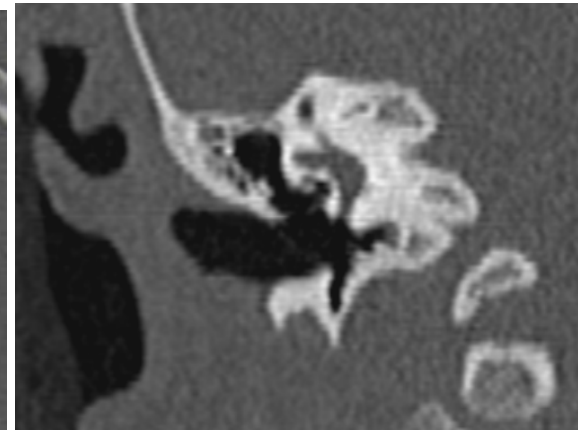

(b)

Figure 11. External auditory meatus in a 3 year old child (A: axial, B: coronal).

\subsection{The Petro-Mastoid Canal}

The petro-mastoid canal is a vestige of the fossa sub arcuata. It connects the posterior fossa and the antrum, passing through the arch formed by the upper semicircular canal and contains a meningeal fold and the subarcuata artery and vein. It appears very wide at birth and gradually fills up during the first year of life due to the growth of the petrous bone [9]. 


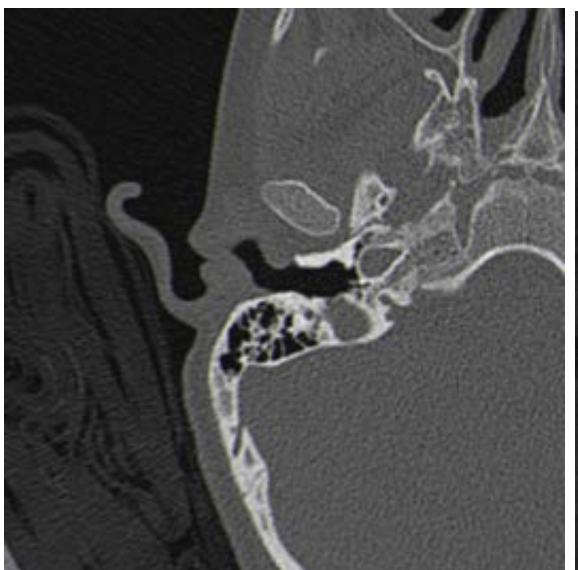

(a)

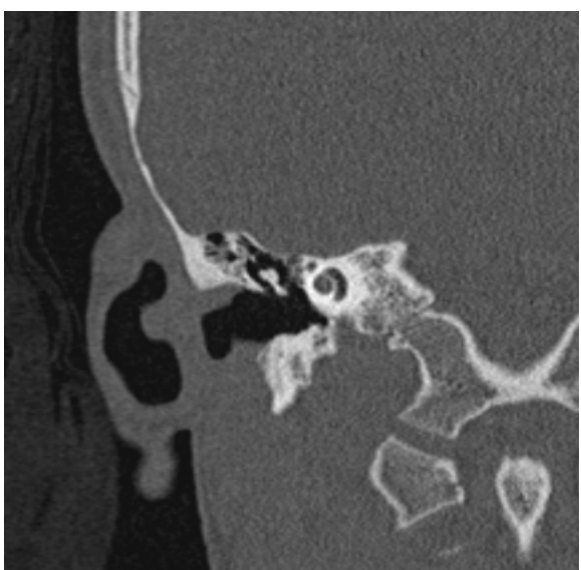

(b)

Figure 12. External auditory meatus in a 14 years old child ((a): axial, (b): coronal).

\subsection{Internal Auditory Meatus (Figure 9)}

The internal auditory meatus is short at birth but lengthens during the first 10 years, more significantly the first year. The diameter of its bottom hardly changes after birth, whereas it can widen discreetly near the porus.

The canal of the cochlear nerve has its definitive size at birth, however, a small diameter is significantly correlated with congenital perception deafness [9].

\subsection{External Auditory Canal}

The external auditory canal is narrow at birth, ossifying gradually [1] (Figures 10-12).

\section{Conclusion}

The petrous bone is a precious tool used widely in pediatrics. This justifies the valorization of an enlightened reading that cannot happen without the perfect knowledge of normal radioanatomy.

\section{References}

[1] Veillon, F. and Jan, C.W. (2013) Imagerie de l'oreille et de l'os temporal. Volume 5, Lavoisier.

[2] Idris, S., Patel, J.H., Renani, A.S., Allan, R. and Vlahos, I. (2015) CT of Normal Developmental and Variant Anatomy of the Pediatric Skull: Distinguishing Trauma from Normality. Radiographics, 35, 1585-1601.

https://doi.org/10.1148/rg.2015140177

[3] Bachor, E., Byahatti, S. and Karmody, S. (1997) The Cochlear Aqueduct in Pediatric Temporal Bone. European Archives of Oto-Rhino-Laryngology, 254, 34-38. https://doi.org/10.1007/BF02439718

[4] Pekkola, J., Pitkaranta, A., Jappel, A., et al. (2004) Localized Pericochlear Hypoattenuating Foci at Temporal-Bone Thin-Section CT in Pediatric Patients: Nonpathologic Differential Diagnostic Entity? Radiology, 230, 88-92.

https://doi.org/10.1148/radiol.2301021111 
[5] Moser, T., Veillon, F., Sick, H. and Riehm, S. (2008) The Hypodense Focus in the Petrous Apex: A Potential Pitfall on Multidetector CT Imaging of the Temporal Bone. AJNR, 29, 35-39. https://doi.org/10.3174/ajnr.A0737

[6] Chadwell, J.B., Halsted, M.J., Choo, D.I., Greinwald, J.H. and Benton, C. (2004) The Cochlear Cleft. $A J N R, 25,21-24$.

[7] Yokoyama, T., Iino, Y., Kakizaki, K. and Murakami, Y. (1999) Human Temporal Bone Study on the Postnatal Ossification Process of Auditory Ossicles. Laryngoscope, 109, 927-930. https://doi.org/10.1097/00005537-199906000-00016

[8] Virapongse, C., Sarwar, M., Bhimani, S., Sasaki, C. and Shapiro, R. (1985) Computed Tomography of Temporal Bone Pneumatization: 1. Normal Pattern and Morphology. AJNR, 6, 551-559. https://doi.org/10.2214/ajr.145.3.473

[9] Elmaleh-Bergès, M., et al. (2006) Le sourd est un enfant: qu'est-ce que ça change. Journal of Radiology, 87, 1795-1812. 